\title{
Resolving Atomic Scale Interfacial Chemical Changes in ODS Ferritic Alloys
}

\author{
J. Aguiar, ${ }^{1,2}$ L. Hsiung, ${ }^{1}$ P. Hosemann,${ }^{3,4}$ M. Fluss, ${ }^{1}$ and N.D. Browning ${ }^{1,2,5}$ \\ ${ }^{1}$ Condensed Matter and Materials Division, Lawrence Livermore National Laboratory, \\ Livermore, CA \\ ${ }^{2}$ Dept. of Chem. Engr. and Matl. Sci., University of California Davis, Davis, CA 95616 \\ ${ }^{3}$ Dept. of Nuclear Engineering, University of California Berkeley, Berkeley, CA 94701 \\ ${ }^{4}$ Los Alamos National Laboratory, P.O. Box 1663 Los Alamos, NM 87545 \\ ${ }^{5}$ Dept. of Molecular Cell Biology, University of California Davis, Davis, CA 95616
}

Since the inception of nuclear power, issues involving nuclear safety and reliability have posed serious practical, financial, security, health and safety problems, due to the heightened pressures, temperatures, and levels of corrosion. Next generation fission and fusion reactors though promise to even surpass conventional nuclear power output and efficiency by increasing the operating temperatures, pressures, and neutron flux over conventional nuclear technology [1]. Concurrently, we have set strict criteria required for safe use of next generation fast reactor and fusion technology [2]. In order therefore meet both heightened operating conditions and strict safety criteria, several materials issues are being investigated at the nanometer to sub-angstrom scale from the minor actinide containing oxide fuel sources to damage resistant steels.

To address the needs of next generation fission and fusion reactor technologies, radiation resistant steels are undergoing strong consideration to address the heightened material demands associated with these emerging extreme environments. In a preliminary effort we have chosen to use aberration corrected STEM [3], electron energy loss spectroscopy (EELS) [4], and atom probe field ion microscopy [5] to unravel the fundamental interfacial structure and chemistry associated with particle-matrix and grain boundary interfaces before and after irradiation, as shown in non-irradiated case for MA-957 in Figure 1, assumed to be primarily responsible for their extended radiation resistance [6].

Extending the study beyond one candidate alloy, we have also chosen to include a comparative study using three-candidate oxide dispersion strengthened (ODS) ferritic alloys [MA-957, K3, PM-2000]. Using the best offerings of microanalysis on each of these candidate alloys, we have focused on developing an understanding based on the observed interfacial structure, composition, and density of interfaces in each alloy to further highlight their extended use in these emerging technologies.

Based on the results in the study, we plan to present a comparitative analysis for each of these three distinctive ODS alloys using the latest microscopy techniques, to develop first level predictions for the best-suited candidate(s) for pending future investigations, that include their nano to macroscale mechanical $[7,8]$ and corrosive properties.

\section{References:}

[1] K. Ehrlich, Philos. Trans. R. Soc. London, Ser. A 357, 595 (1999).

[2] E. E. Bloom, S. J. Zinkle, and F. W. Wiffen, J. Nucl. Mater. 329-333, 12 (2004). 
[3] Batson P.E., Delby N, and Krivanek O.L. , Nature 418617 (2002).

[4] Egerton R F Electron Energy-Loss Spectroscopy in the Electron Microscope 2nd edn (New York: Plenum/Springer) (1996)

[5] Miller M K, 1996 Atom Probe Field Ion Microscopy (New York: Oxford University Press)

[6] S. Ukai and M. Fujiwara, J. Nucl. Mater. 307-311, 749 (2002).

[7] P. Hosemann, et. al. , J. Nucl. Mater. 375323 (2008).

[8] P. Hosemann et. al., J. Nucl. Mater. doi: 10.1016/ j.jnucmat.2010.12.200 (2010)

[9] The authors acknowledge support from the Lawrence Livermore Graduate Scholar Program. This work was performed under the auspices of the U.S. Department of Energy by Lawrence Livermore National Laboratory under Contract DE-AC52-07NA27344.

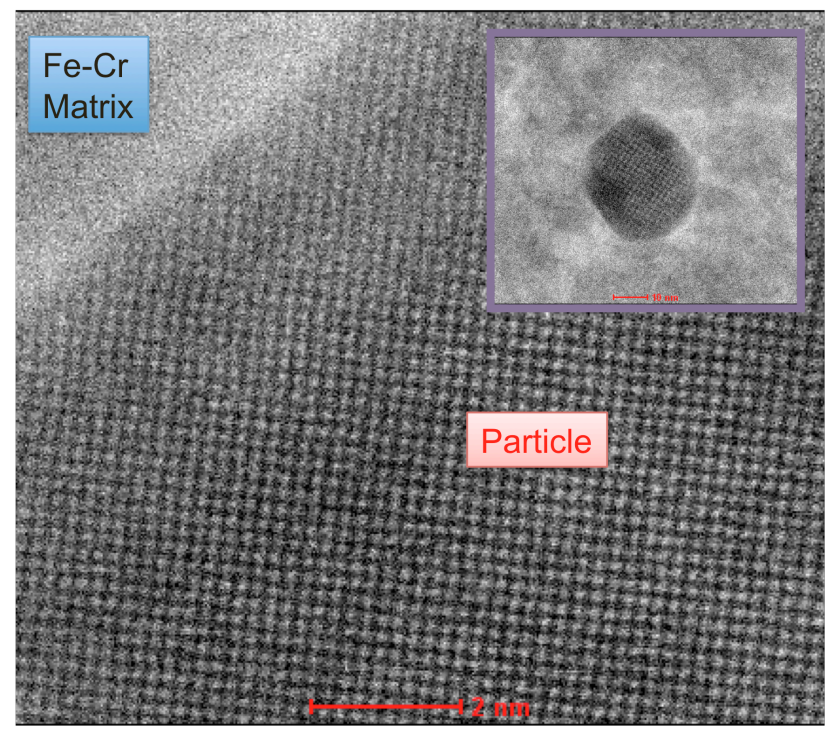

Figure 1: Unprocessed aberration corrected HR-STEM image of the incoherent interface of an embedded yttria oxide particle in the ironchromium matrix for non-irradiated K3 alloy sample. 